TRANSACTIONS OF THE

AMERICAN MATHEMATICAL SOCIETY

Volume 195, 1974

\title{
ON FREE PRODUCTS OF FINITELY GENERATED ABELIAN GROUPS(1)
}

BY

\section{ANTHONY M. GAGLIONE}

ABSTRACT. Let the group $G$ be a free product of a finite number of finitely generated abelian groups. Let $G$ ' be its commutator subgroup. It is proven here that the "quasi-G-simple" commutators, defined below, are free generators of $G^{\prime}$.

I. Introduction. In this paper, we will investigate the commutator subgroup, $G^{\prime}$, of a group, $G$, which is the free product:

$$
G=G(1) * G(2) * \cdots * G(s)
$$

where each $G(i)$ for $i=1,2, \cdots, s$ is a finitely generated abelian group. We observe that the Kurosh subgroup theorem [5, p. 243] implies that $G^{\prime}$ is a free group. Using a Kurosh rewriting process in the manner of [1], we obtain a set of free generators for $G^{\prime}$. Then by means of the commutator calculus and a wellknown theorem on free groups, we produce in Theorem 2.1 a more useful set of free generators of $G$ ", the "quasi-G-simple" commutators.

The results obtained here are generalizations of those in [1], [2], [3], [6], [9]. In [3], K. W. Gruenberg found a set of free generators for the commutator subgroup of a group which is the free product of finitely many cyclic groups. We note that the "quasi-G-simple" commutators become identical with the generators of Theorem $5.2[3]$ in the special case where $G$ is a free product of cyclic groups. In [2], S. Bachmuth studied the commutator subgroup of a free metabelian group with finitely many free generators. R. Prener, in [6], found free generators for the commutator subgroup of a group which is the free product of direct products of cyclic groups of order two. In [9], H. V. Waldinger generalizes this from two to

Presented to the Society, April 21, 1973 under the title Lower central series of free products of finitely generated abelian groups; received by the editors March 23, 1973.

AMS (MOS) subject classifications (1970). Primary 20E30, 20F35, 20K05; Secondary 20F05, 20F 15 .

Key words and phrases. Free product of groups, free group, finitely generated abelian group, free generator, basic commutator, commutator subgroup, dimension, " $G$ simple basic commutator," "quasi-G-simple" commutator, rewriting process.

(1) This paper forms part of the author's doctoral dissertation written at the Polytechnic Institute of Brooklyn, under the guidance of H. V. Waldinger. 
any prime order by adapting the methods of [2]. Moreover, the "quasi-G-simple" commutators reduce to the " $G$-simple basic commutators" of Theorem 2 [9] in the special case where each $G(i)$ is the direct product of cyclic groups of prime order. Finally, a set of free generators for the commutator subgroup of a group which is the free product of finite abelian groups is found in [1] by means of the Kurosh rewriting process. The set, $\Sigma$, of free generators of $G^{\prime}$ found in Lemma 3.3 of this paper reduces to the corresponding set found in [1] if the free factors of $G$ are finite abelian groups.

To proceed we shall employ the terminology and notation of $\$ \$ 2$ and 3 of [8]. [8] shall be referred to as I. Furthermore, the numbering of any theorem, definition, or lemma in I will be denoted by a.b-I; e.g., Lemma 2.1-I shall mean Lemma 2.1 of $\mathrm{I}$.

II. Statement of the main result. We first require two definitions.

Definition 2.1. (For a comparison see Definition 4.1-I.) A basic commutator (refer to Definition 2.1-I), $c$, is called G-simple if it satisfies four conditions:

(i) Either $c$ is among the generators $c_{1}, c_{2}, \cdots, c_{r}$ described in $\$$ III below, or $c=\left(\cdots\left(c_{j_{1}}, c_{j_{2}}\right), \cdots, c_{j_{\omega}}\right)$ such that $c_{r} \geq c_{j_{1}}>c_{j_{2}} \geq c_{1}$ and $c_{j_{2}} \leq c_{j_{3}} \leq \cdots$ $\leq c_{j_{\omega}} \leq c_{r}$

(ii) If $D(c)>1$, then $\left(c_{j_{1}}, c_{j_{2}}\right) \neq 1$ in $G$. of $c_{i^{*}}$ )

(iii) If $c_{i}$ occurs $\sigma$-times in $\langle c\rangle$, then $1 \leq \sigma<O\left(c_{i}\right)$. (O(c $\left.c_{i}\right)$ means the order

(iv) If $D(c)>2$, and $\left(c_{j_{1}}, c_{j_{\tau}}\right)=1$ in $G$ for $2<\tau \leq \omega$ then $c_{j_{\tau}} \leq c_{j_{1}}$.

Definition 2.2. Let $t>1$. A commutator

$$
e=\left(\cdots\left(c_{i_{1}}^{\epsilon}, c_{i_{2}}^{\epsilon}\right), \cdots, c_{i_{t}}^{t}\right)
$$

is a quasi-G-simple commutator provided

(a) $c=\left(\cdots\left(c_{i_{1}}, c_{i_{2}}\right), \cdots, c_{i_{t}}\right)$ is G-simple,

(b) the $\epsilon_{j}= \pm 1$ for $j=1,2, \cdots, t$,

(c) $\epsilon_{j}=-1$ only if $O\left(c_{i_{j}}\right)=\infty$,

(d) if $\epsilon_{j}=-1$, then $\epsilon_{k}=-1$ for all $c_{i_{k}}=c_{i_{j}}$.

Theorem 2.1. The quasi-G-simple commutators are free generators of $G^{\prime}$.

III. A set of free generators of $G^{\prime}$. It is well known that any $G(i)$ in $(1.1)$ is the direct product of cyclic groups of either prime power or infinite order. (See [4].) Thus we let $G$ have generators $c_{1}, c_{2}, \cdots, c_{r}$ with prime power or infinite order. Let $j=1,2, \cdots, r$. Let $a_{j}=O\left(c_{j}\right)$ if $O\left(c_{j}\right)$ is finite but let $a_{j}=0$ if $O\left(c_{j}\right)=\infty$. 
To arrive at our main result, Theorem 2.1, we first obtain a set, $\Sigma$, of free generators of $G^{\prime}$ in Lemma 3.3. This set, $\Sigma$, is obtained by applying the Kurosh rewriting process $[5, \$ 4.3]$ to the commutator subgroup, $G^{\prime}$, of $G$. This procedure starts out by dividing the generators of $G$ into disjoint classes.

It will be understood that the generators $c_{1}, c_{2}, \cdots, c_{r}$ are ordered by their subscripts so that for $1 \leq i<j \leq s$ a generator of $G(i)$ always precedes a generator of $G(j)$. Clearly there exist integers

$$
0=n_{0}<n_{1}<n_{2}<\cdots<n_{s}=r
$$

so that

$$
c_{n_{i-1+1}^{\prime}}, c_{n_{i-1+2}}, \cdots, c_{n_{i}}
$$

generate $G(i)$ for $i=1,2, \cdots, s$. We shall say that the generators given in (3.2) constitute the class $\beta_{i}$ or are generators of $\beta_{i}$-type.

Having defined the classes $\beta_{i}$, we must next introduce coset representatives of $\beta_{1} \cdot, \beta_{2}, \cdots, \beta_{s}-$, and neutral types. To construct these representatives, we require Criterion 1 below which involves quantities $\sigma(k, w)$. Any nonidentity element, $w$, of $G$ evidently has the form

$$
w=\prod_{i=1}^{\tau} c_{j_{i}}^{\epsilon_{i}}
$$

where the $\epsilon_{i}$ are integers and are such that (i) $0<\epsilon_{i}<\alpha_{j_{i}}$ if $\alpha_{j_{i}} \neq 0$, (ii) at least one among $\epsilon_{1}, \epsilon_{2}, \cdots, \epsilon_{\tau} \neq 0$. Let $k=1,2, \cdots, r$. For $w \neq 1$, we then take

$$
\sigma(k, w)= \begin{cases}\sum_{j_{i}=k} \epsilon_{i} & \text { if } k \in K=\left\{j_{1}, j_{2}, \cdots, j_{\tau}\right\}, \\ 0 & \text { if } k \notin K .\end{cases}
$$

For $w=1$, we let every $\sigma(k, w)=0$.

As an immediate consequence of the well-known fact [5, p. 79] that a word $w$ in the free group on $r$ generators is in the commutator subgroup if and only if every generator occurs in $w$ with "exponent sum" zero, we now easily obtain our criterion.

Criterion 1. $w \in G^{\prime}$ if and only if the $r$ quantities $\sigma(k, w)$ are either (i) divisible by $a_{k}$ if $a_{k} \neq 0$, or (ii) $=0$ if $a_{k}=0$.

Our criterion clearly implies

Lemma 3.1. The words $w_{1}$ and $w_{2}$ are in the same coset of $G^{\prime}$ if and only if each of the $r$ differences $\sigma\left(k, w_{1}\right)-\sigma\left(k, w_{2}\right)$ is either divisible by $\alpha_{k}$ if $\alpha_{k} \neq 0$ or $=0$ if $a_{k}=0$. 
Making use of this lemma, we can now obtain many systems of coset representatives. In order to arrive at the desired set of free generators of $G^{\prime}$, however, we shall choose the coset representatives in the special manner given below. Using a scheme analogous to that of [1], we proceed as follows:

Let the $\beta_{i}$-representatives $(1 \leq i \leq s)$ be given by

$$
K_{i}\left(\epsilon_{1}, \cdots, \epsilon_{r}\right)=\left\{\begin{array}{l}
\prod_{b=0}^{s-1}\left[\prod_{j=n_{s-b-1}}^{n_{s}-b} c_{j}^{\epsilon_{j}}\right] \text { for } i=1 \\
{\left[\prod_{b=0, b \neq s-i}^{s-1}\left[\prod_{j=n_{s-b-1}}^{n_{s-b}} c_{j}^{\epsilon_{j}}\right]\right]\left[\prod_{j=n_{i-1+1}}^{n_{i}} c_{j}^{\epsilon_{j}}\right] \text { for } i>1}
\end{array}\right.
$$

where

$$
0 \leq \epsilon_{j}<\alpha_{j} \text { if } \alpha_{j} \neq 0 .
$$

Let the representatives of neutral and $\beta_{1}$-types be identical.

For fixed $i$, it is obvious by Lemma 3.1 that the group elements $K_{i}\left(\epsilon_{1}, \cdots, \epsilon_{r}\right)$ are in distinct cosets of $G^{\prime}$. According to [1] and [5, p. 239], this collection of coset representatives forms a regular extended Schreier system.

Having verified that our representative system is an extended Schreier system (we do not need the fact that it is regular), we immediately find a set of free generators of $G^{\prime}$ by making use of

Lemma 3.2. (See Corollary 4.8 of [5].) Let $G$ be a free product of the form (1.1). Let $H$ be a subgroup of $G$. For $w \in G$ let ${ }^{*} w$ and $\beta_{i}(w)$ be the representatives of $w$ of neutral and $\beta_{i}$-type respectively. Let $t_{N}=N\left({ }^{*} N\right)^{-1}$ where $N$ is a right coset representative of any $\beta_{i}$-type. Let $s_{N, x}=N x\left(\beta_{i}(N x)\right)^{-1}$ where $N$ is a right coset representative and $x$ is a generator both of the same $\beta_{i}$-type. Suppose the coset representatives of $\beta_{1}^{-}, \beta_{2}^{-}, \cdots, \beta_{s^{-}}$, and neutral types constitute an extended Schreier system. Then $H$ is the free product $H=H_{1} * H_{2}$ where $H_{1}$ has as free generators those $t_{N}$ which are $\neq 1$ and $H_{2}$ is generated by elements $s_{N_{0} x^{*}}$

Let us apply Lemma 3.2 to our case. (Here $G^{\prime}$ is normal and we do not distinguish between right and left cosets.) If $x$ is a generator and $N=$ $K_{i}\left(\epsilon_{1}, \epsilon_{2}, \cdots, \epsilon_{\tau}\right)$ is a coset representative both of $\beta_{i}$-type, then $N x$ is evidently again a coset representative of the form (3.3) since the generators of $G(i)$ commute and have either prime power or infinite order. Thus Lemma 3.2 yields

Lemma 3.3. The following set $\Sigma \subseteq G^{\prime}$ is a set of free generators for $G^{\prime} . \Sigma$ consists of all elements of the form 


$$
t_{i}\left(\epsilon_{1}, \cdots, \epsilon_{r}\right)=\left[K_{i}\left(\epsilon_{1}, \cdots, \epsilon_{r}\right)\right]\left[K_{1}\left(\epsilon_{1}, \cdots, \epsilon_{r}\right)\right]^{-1}
$$

which are such that (i) $i \neq 1$, (ii) at least one $\epsilon_{j} \neq 0$ for $\left(n_{i-1}+1\right) \leq j \leq n_{i}$, and finally (iii) at least one $\epsilon_{j} \neq 0$ for $1 \leq j \leq n_{i-1}$.

Next by means of the commutator calculus and a well-known theorem on free groups, we proceed to show our main result, Theorem 2.1, which we restate here.

Theorem 2.1. The quasi-G-simple commutators are free generators of the same group as $\Sigma$. In other words, the quasi-G-simple commutators generate $G^{\prime}$ freely.

To prove this theorem we shall first set up a one-to-one correspondence between the elements of the set $\Sigma(3.5)$ and the quasi-G-simple commutators. For this purpose we need to introduce the auxiliary quantities below.

$$
\text { Let } \eta_{j}= \begin{cases}0 & \text { if } \epsilon_{j}=0 \\ \alpha_{j}-\epsilon_{j} & \text { if } \epsilon_{j} \neq 0\end{cases}
$$

$$
\text { Let } E=E\left(\epsilon_{1}, \epsilon_{2}, \cdots, \epsilon_{r}\right)=\sum_{j=1}^{r}\left|\eta_{j}\right| \cdot
$$

Let $\sigma=\sigma\left(i ; \epsilon_{1}, \epsilon_{2}, \cdots, \epsilon_{r}\right)$ be the largest integer among $n_{i-1}+1, n_{i-1}+2, \cdots$, $n_{i}$ so that $\epsilon_{\sigma} \not 0$. ( $\sigma$ can always be found uniquely by condition (ii) on the elements of $\Sigma_{\text {.) }}$

Finally let $\lambda_{1}, \lambda_{2}, \cdots, \lambda_{E}$ be that sequence of integers such that

(i) $\lambda_{1}=\sigma\left(i ; \epsilon_{1}, \epsilon_{2}, \cdots, \epsilon_{r}\right)$;

(ii) $\lambda_{2} \leq \lambda_{3} \leq \cdots \leq \lambda_{E}$, when $E>2$;

(iii) any $j$ among $1,2, \ldots, r$ occurs $\left|\eta_{j}\right|$ times among $\lambda_{1}, \lambda_{2}, \cdots, \lambda_{E^{*}}$

We have now developed the machinery which we require for our 1-1 correspondence. We define

$$
c\left(i ; \epsilon_{1}, \epsilon_{2}, \cdots, \epsilon_{1}\right)=\left(\cdots\left(c_{\lambda_{1}}^{\gamma_{1}}, c_{\lambda_{2}}^{\gamma_{2}}\right), \cdots, c_{\lambda_{E}}^{\gamma_{E}}\right)
$$

where $\gamma_{i}=1$ if $\eta_{\lambda_{i}}>0$ and $\gamma_{i}=-1$ if $\eta_{\lambda_{i}}<0$.

Since an element of the set $\Sigma$ is such that at least one $\epsilon_{j} \neq 0$ for $1 \leq j \leq$ $n_{i-1}$, it follows from (3.1), (3.2), (3.8), and (3.9) and Definition 2.1-I that (i) $\left(c_{\lambda_{1}}{ }_{1}^{1}, c_{\lambda_{2}}^{\gamma_{2}}\right) \neq 1$ in $G$, and (ii) any generator $c_{k}$ which is in the set, 
$\left\{c_{\lambda_{2}}, c_{\lambda_{3}}, \cdots, c_{\lambda_{E}}\right\}$, and is of the same type as $c_{\lambda_{1}}$ is also $\leq c_{\lambda_{1}}$. Furthermore by Definitions 2.1 and 2.2, equations (3.6) through (3.10), and the inequalities (3.4), we find that $c\left(i ; \epsilon_{1}, \epsilon_{2}, \cdots, \epsilon_{r}\right)$ is indeed a quasi-G-simple commutator.

Conversely, given any quasi-G-simple commutatoc $c=\left(\ldots\left(c_{\lambda_{1}}^{\gamma_{1}}, c_{\lambda_{2}}^{\gamma_{2}}\right), \cdots, c_{\lambda_{E}}^{\gamma_{E}}\right)$ we easily find a unique

$$
t(c)=t_{i}\left(\epsilon_{1}, \epsilon_{2}, \cdots, \epsilon\right)
$$

so that (3.11) holds for the integers $i, \epsilon_{1}, \epsilon_{2}, \cdots, \epsilon_{r}$ in (3.12). We do this by choosing $i$ so that $c_{\lambda_{1}}$ is of $\beta_{i}$-type and by reversing the above procedure for finding $\lambda_{1}, \lambda_{2}, \cdots, \lambda_{E}$ as functions of $\epsilon_{1}, \epsilon_{2}, \cdots, \epsilon_{r}$. Hence by Definitions 2.1 and 2.2 , it is clear that we must be led to a unique member of the set $\Sigma$.

Next we require an ordering of the quasi-G-simple commutators. We note that the $G$-simple basic commutators are ordered by the ordering for basic commutators in Definition 2.1-I.

We shall call the quasi-G-simple commutators

$$
d_{1}=\left(\ldots\left(c_{i_{1}}^{\epsilon_{1}}, c_{i_{2}}^{\epsilon_{2}}\right), \ldots, c_{i_{\theta}}^{\epsilon_{\theta}}\right) \text { and } d_{2}\left(\ldots\left(c_{j_{1}}^{\delta_{1}}, c_{j_{2}}^{\delta_{2}}\right), \ldots, c_{j_{\tau}}^{\delta_{\tau}}\right)
$$

equivalent if and only if (i) $\theta=r$ and (ii) $i_{k}=j_{k}$ for $1 \leq k \leq \theta$. This clearly constitutes an equivalence relation so that the quasi-G-simple commutators are broken up into disjoint equivalence classes. In each class $S_{\rho}$, pick out $c_{\rho} \in S_{\rho}$ where $c_{\rho}$ is the $G$-simple basic commutator in $S_{\rho}$. We will say

$$
S_{\rho_{1}}<S_{\rho_{2}}
$$

if and only if $\rho_{1} \neq \rho_{2}$ and $c_{\rho_{1}}<c_{\rho_{2}}$. Any quasi-G-simple commutator in a given class follows all those in earlier classes in the ordering (3.13). Those quasi-Gsimple commutators in the same class are ordered arbitrarily with respect to each other. Quasi-G-simple commutators will always be numbered so that they are ordered by their subscripts.

In order to establish that the quasi-G-simple commutators generate $G^{\prime}$ freely, we need the following preliminary lemma:

Lemma 3.4. Consider the quasi-G-simple commutator $c=\left(\cdots\left(c_{\lambda_{1}}^{\gamma_{1}}, c_{\lambda_{2}}^{\gamma_{2}}\right), \cdots, c_{\lambda_{E}}^{\gamma_{E}}\right)$. Then

$$
t(c)=w_{1} c^{-1} w_{2}
$$

where $w_{1}$ and $w_{2}$ are either both 1 or they are in the subgroup generated by those quasi-G-simple commutators which are in classes earlier than $c$ in the ordering (3.13). 
Proof. We observe that it clearly suffices to prove this lemma for the case in which all $\gamma_{i}(1 \leq i \leq E)$ are positive. For suppose some $\gamma_{i}=-1$. Then we may replace the generator $c_{\lambda_{i}}$ by $d_{\lambda_{i}}=c_{\lambda_{i}}^{-1}$ and work with this set of generators. (Note that this is justified by virtue of condition (d) in Definition 2.2.) Thus without lack of generality, we assume that $c$ is G-simple.

Suppose that $j(r)$ is such that $c_{\lambda_{T}}$ is of $\beta_{j(r)^{\text {type }}}$ where $1 \leq \tau \leq E$. We shall treat the following three cases:

I. $j(r)<j(1)$ for $2 \leq r \leq E$,

II. $j(r) \leq j(1)$ for $2 \leq r \leq E$,

III. $j(r)>j(1)$ for some $r$ which is $>2$ but $\leq E$.

Case I. Let us first analyze Case I by induction on $E$. For $E=2$, we easily find by our 1-1 correspondence that

$$
t(c)=c_{\lambda_{2}}^{-1} c_{\lambda_{1}}^{-1} c_{\lambda_{2}} c_{\lambda_{1}}=c^{-1} \text {. }
$$

To proceed we then make the induction hypothesis that the lemma holds in the present Case I for $2 \leq E<\bar{E}$ so that $w_{1}$ and $w_{2}$ have the following property $\mathcal{L}$.

Property $\mathcal{L} . w_{1}$ and $w_{2}$ are either both 1 or are in the subgroup generated by those $G$-simple basic commutators of the form $d=\left(\cdots\left(c_{\rho_{1}}, c_{\rho_{2}}\right), \cdots, c_{\rho_{\theta}}\right)$ which are such that (i) $\left\{\rho_{1}, \rho_{2}, \cdots, \rho_{\theta}\right\}$ is a proper subset of $\left\{\lambda_{1}, \lambda_{2}, \cdots, \lambda_{E}\right\}$, (ii) $\rho_{\theta} \leq \lambda_{E}$ and (iii) if $j(E)<j(1), \rho_{1}$ must $=\lambda_{1}$. (By Definition 2.1-I, every such $d$ is clearly $<c_{0}$ )

Having made our hypothesis and noting that property $\mathcal{L}$ holds trivially for $E=2$, we are ready to consider $E=\bar{E}$. Our $1-1$ correspondence yields now

$$
\begin{aligned}
(t(c))^{-1} & =c_{\lambda_{1}}^{-1}\left[\prod_{k=0}^{E-2}\left(c_{\lambda_{E-k}}\right)^{-1}\right] c_{\lambda_{1}}\left[\prod_{k=0}^{E-2} c_{\lambda_{2}+k}\right] \\
& =\left(c_{\lambda_{1}}, c_{\lambda_{E}}\right) c_{\lambda_{E}^{-1}\left(t\left(c^{L}\right)\right)^{-1} c_{\lambda_{E}}}
\end{aligned}
$$

By application of the induction hypothesis and of the trivial identity

$$
b^{-1}\left(\prod_{i=1}^{I} a_{i}^{\epsilon_{i}}\right) b=\prod_{i=1}^{I}\left[a_{i}\left(a_{i}, b\right)\right]^{\epsilon_{i}}
$$

and by virtue of Definitions 2.1 and 2.1-I, we then can express $c$ in the form (3.14) so that $w_{1}$ and $w_{2}$ have Property $\mathscr{L}$.

Having carried out the desired proof for Case I, we are now ready to go to Case II. Our 1.1 correspondence now expresses $t(c)$ as

$$
(t(c))^{-1}=\left(c_{\lambda_{1}} u\right)^{-1}\left(\prod_{k=0}^{\mu-2} c_{\lambda_{\mu-k}}^{-1}\right)\left(c_{\lambda_{1}} u\right)\left(\prod_{k=0}^{\mu-2} c_{\lambda_{2}+k}\right)
$$


so that (i) $\left(\Pi_{k=0}^{\mu-2} c_{\lambda_{2+k}}\right)$ is the product of those generators in the set $\Gamma=$ $\left\{c_{\lambda_{2}}, c_{\lambda_{3}}, \cdots, c_{\lambda_{E}}\right\}$ which are such that $j(\tau)<j(1)$ for $2 \leq \tau \leq \mu$,

(ii) $u=1$ if $\Gamma$ does not contain generators in the class $j(1)$, but is the product $u=\Pi_{k=1}^{E-\mu} c_{\lambda_{\mu+k}}$ of those generators in $\Gamma$ which are in class $j(1)$ if there are such generators. We note from the hypothesis of this case that $\Gamma$ does not contain generators in classes $\beta_{m}$ where $m>j(1)$. Proceeding by induction on $E-\mu$, we shall now prove the truth of $(3.14)$ in Case II so that $w_{1}$ and $w_{2}$ have property $\mathcal{L}$. For $E-\mu=0$ we have already done so in Case I. Suppose then that we have shown the desired conclusion for $\mu \leq E<\bar{E}$. We consider $E=\bar{E}$ next and rewrite (3.16) as

$$
\begin{aligned}
\left(t_{t}(c)\right)^{-1}= & \left\{c_{\lambda_{E}}^{-1}\left[\left(c_{\lambda_{1}} u c_{\lambda_{E}}^{-1}\right)^{-1}\left[\prod_{k=0}^{\mu-2}\left(c_{\lambda_{\mu-k}}\right)^{-1}\right]\left(c_{\lambda_{1}} u c_{\lambda_{E}}^{-1}\right)\left[\prod_{k=0}^{\mu-2} c_{\lambda_{2+k}}\right] c_{\lambda_{E}}\right\}\right. \\
& \cdot\left\{\left(c_{\lambda_{E}}, \prod_{k=0}^{\mu-2} c_{\lambda_{2+k}}\right)\right\} \\
= & \left(c_{\lambda_{E}}^{-1} t\left(c^{L}\right)^{-1} c_{\lambda_{E}}\right)\left(\left(c_{\lambda_{E}}, \prod_{\dot{k}=0}^{\mu-2} c_{\lambda_{2+k}}\right)\right) .
\end{aligned}
$$

We now apply the induction hypothesis to $t\left(c^{L}\right)$, and we make use of (3.15) to rewrite $c_{\lambda_{E}}^{-1}\left(t\left(c^{L}\right)\right)^{-1} c_{\lambda_{E}}$ and then express it as a product of $G$-simple basic commutators by Lemma 4.5-I. (We note that in I the generators were assumed to have prime order, but the proof of this lemma goes through exactly the way it stands in our case where the generator $c_{j}$ either has $O\left(c_{j}\right)=a_{j}$ or $=\infty_{\text {.) }}$ We also find by repeated reference to the well-known identity $(a, b c)=(a, c)(a, b)((a, b), c)$ and to (3.15) that $\left(c_{\lambda_{E}}, \Pi_{k=0}^{\mu-2} c_{\lambda_{2+k}}\right)$ is a word in commutators of the form

$$
\left(\cdots\left(\left(c_{\lambda_{E}}, c_{\eta_{1}}\right), c_{\eta_{2}}\right), \cdots, c_{\eta_{\phi}}\right),
$$

where $\eta_{1}, \eta_{2}, \cdots, \eta_{\phi}$ is a subsequence of $\lambda_{2} \leq \lambda_{3} \leq \cdots \leq \lambda_{\mu}$. It is clear by Definitions $2.1-\mathrm{I}$ and 2.1 that this procedure must give us (3.14) once again so that $w_{1}$ and $w_{2}$ have property $\mathcal{L}$.

Having proven the lemma for Cases I and II, we now complete the proof by considering

Case III. Our 1-1 correspondence gives us $(t(c))^{-1}=a^{-1} \Omega \alpha$ for this final case, where $\Omega$ is given by the right-hand side of (3.16) and $\alpha$ is the product, $\alpha=\Pi_{k=\widetilde{E}+1}^{E} c_{\lambda_{k}}$, of those elements of $\Gamma$ which are in classes $\beta_{m}$ such that $m>j(1)$. We then express $\Omega$ as in Case II and apply the identity (3.15) repeatedly. 
Recalling Definitions 2.1-I and 2.1 we now verify the truth of our lemma also for this last case.

At this point we are ready to apply Lemma 3.4 and thus proceed to the

Proof of Theorem 2.1. Let $d_{1}, d_{2}, \cdots, d_{n}, \cdots$ be the quasi-G-simple commutators ordered in the manner described after inequality $(3.13)$. Let $t^{(1)}, t^{(2)}, \ldots$, $t^{(n)}, \ldots$ be the corresponding generators of the set $\Sigma$ found by (3.12). Taking $\tau=1,2, \cdots, n, \cdots$ we conclude by $(3.14)$ that $d_{1}, d_{2}, \cdots, d_{n}, \cdots$ generate the same group as the set $\Sigma$. To show that $d_{1}, d_{2}, \cdots, d_{n}, \cdots$ generate $G^{\prime}$ freely, let us suppose that there were a nontrivial relation

$$
R=R\left(d_{\rho_{1}}, d_{\rho_{2}}, \cdots, d_{\rho_{e}}\right)=1
$$

among the quasi-G-simple commutators. Let $M$ be the maximum among $\rho_{1}, \rho_{2}, \cdots$, $\rho_{e}$. We note that

$$
\Lambda=\left\{t^{(1)}, t^{(2)}, \ldots, t^{(M)}\right\}
$$

is a subset of the set, $\Sigma$, of free generators. Hence the elements of $\Lambda$ are free generators of the group which they generate. But this implies that $d_{1}, d_{2}, \cdots, d_{M}$ are free generators according to the well-known Theorem 3.1, below, a fact which contradicts the relation $R=1$. Therefore we have shown that the quasi-G-simple commutators generate $G^{\prime}$ freely.

Theorem 3.1 ([5, p. 110] or [4, p. 109]). If a free group has $q<\infty$ free generators, then any $q$ generators are free generators.

\section{REFERENCES}

1. M. Anshel and R. Prener, On free products of finite abelian groups, Proc. Amer. Math. Soc. 34 (1972), 343-345. MR $46 \# 1911$.

2. S. Bachmuth, Automorphisms of free metabelian groups, Trans. Amer. Math. Soc. 118 (1965), 93-104. MR 31 \#4831.

3. K. W. Gruenberg, Residual properties of infinite soluble groups, Proc. London Math. Soc. (3) 7 (1957), 29-62. MR 19, 386.

4. M. Hall, Jro, The theory of groups, Macmillan, New York, 1959. MR 21 \#1996.

5. W. Magnus, A. Karass and D. Solitar, Combinatorial group theory: Presentations of groups in terms of generators and relations, Pure and Appl. Math., vol. 13, Interscience, New York, 1966. MR 34 \#7617.

6. R. A. Prener, The lower central series of special groups generated by elements of order two, Ph.D. Thesis, The Polytechnic Institute of Brooklyn, Brooklyn, N. Y., 1969.

7. H. V. Waldinger, Addendum to "The lower central series of groups of a special class", J. Algebra 25 (1973), 172-175. 
8. H. V. Waldinger, The lower central series of groups of a special class, J. Algebra 14 (1970), 229-244. MR 41 \#5502.

9. - Two theorems in the commutator calculus, Trans. Amer. Math. Soc. 167 (1972), 389-397. MR 45 \#3537.

DEPARTMENT OF MATHEMATICS, CITY COLLEGE (CUNY), NET YORK, NEW YORK 10032 